\title{
Assessing Bone Marrow Activity in Patients with Myelofibrosis: Results of a Pilot Study of ${ }^{18}$ F-FLT PET
}

\author{
Laetitia Vercellino $^{1}$, Matthieu John Ouvrier ${ }^{2}$, Emmanuelle Barré1, Bruno Cassinat ${ }^{3}$, Virginie de Beco ${ }^{2}$, \\ Christine Dosquet $^{3}$, Sylvie Chevret ${ }^{4,5}$, Véronique Meignin ${ }^{6}$, Christine Chomienne ${ }^{3,5}$, Marie-Elisabeth Toubert ${ }^{1}$, \\ Pascal Merlet ${ }^{1,5}$, and Jean-Jacques Kiladjian ${ }^{5,7}$
}

${ }^{I}$ Service de Médecine Nucléaire, Hôpital Saint-Louis, Assistance-Publique Hôpitaux de Paris, Paris, France; ${ }^{2}$ Service de Médecine Nucléaire, Hôpital Avicenne, Assistance-Publique Hôpitaux de Paris, Bobigny, France; ${ }^{3}$ Unité de Biologie Cellulaire, Hôpital SaintLouis, Assistance-Publique Hôpitaux de Paris, Paris, France; ${ }^{4}$ Service de Biostatistique et Information Médicale, Hôpital Saint-Louis, Assistance-Publique Hôpitaux de Paris, Paris, France; ${ }^{5}$ Université Paris-Diderot (Paris 7), Paris, France; ${ }^{6}$ Service d'AnatomoPathologie, Hôpital Saint-Louis, Assistance-Publique Hôpitaux de Paris, Paris, France; and ${ }^{7}$ Centre d'Investigations Cliniques, Hôpital Saint-Louis, Assistance-Publique Hôpitaux de Paris France, Paris, France

An emerging noninvasive approach to assess tissue proliferation uses the PET tracer $3^{\prime}$-deoxy-3'-18 F-fluorothymidine $\left({ }^{18} \mathrm{~F}-\mathrm{FLT}\right)$. To evaluate the diagnostic value of this technique in myelofibrosis, ${ }^{18} \mathrm{~F}-\mathrm{FLT}$ PET imaging results were compared with bone marrow histology and bone marrow scintigraphy (BMS), the gold standard techniques in this clinical situation. Methods: Fifteen patients with histology-proven myelofibrosis were included consecutively in the study. Tracers' distributions were assessed using a visual grading assessment score of the uptake in the axial skeleton, proximal and distal limbs, liver, and spleen. This visual score was used to define patterns of tracer distribution and to compare the information provided either by PET or by BMS. A semiquantitative analysis with determination of $S_{U} V_{\max }$ in the same localizations was performed for ${ }^{18} \mathrm{~F}-\mathrm{FLT}$ PET. Results: The histology grade of fibrosis correlated with the SUV $\mathrm{max}_{\text {ax }}$ in the axial skeleton (spine and iliac crests) and proximal limbs. ${ }^{18} \mathrm{~F}$-FLT uptake in these areas was much lower in patients with grade 3 fibrosis than in patients with grade 1 or 2 fibrosis. ${ }^{18} \mathrm{~F}-\mathrm{FLT}$ PET showed the same distribution of uptake as BMS in 13 of 14 patients (1 patient did not undergo BMS). In 1 patient, ${ }^{18} \mathrm{~F}-\mathrm{FLT}$ PET clearly showed an intense abnormal splenic uptake, whereas spleen uptake was inconclusive with BMS. Conclusion: ${ }^{18} \mathrm{~F}-\mathrm{FLT}$ PET appears to be a reliable and convenient technique to assess hematopoietic activity in bone marrow. It yields results close to those observed with BMS. In our study population, ${ }^{18} \mathrm{~F}-\mathrm{FLT}$ uptake in the axial skeleton and proximal limbs assessed by SUV $\mathrm{V}_{\max }$ correlated with the grade of fibrosis. Thus, ${ }^{18} \mathrm{~F}-\mathrm{FLT}$ PET may be a useful tool to measure the severity of myelofibrosis, and to monitor noninvasively the patients' status during follow-up. Finally, ${ }^{18} \mathrm{~F}$-FLT PET may be foreseen as an alternative to BMS.

Key Words: ${ }^{18} \mathrm{~F}-\mathrm{FLT}$; PET; myelofibrosis; bone marrow imaging

J Nucl Med 2017; 58:1603-1608

DOI: 10.2967/jnumed.116.188508

$\mathbf{M}$ yeloproliferative neoplasm-associated myelofibrosis includes 3 clinical-pathologic entities: primary myelofibrosis diagnosed

Received Dec. 28, 2016; revision accepted Mar. 20, 2017.

For correspondence or reprints contact: Laetitia Vercellino, Service de Médecine Nucléaire Hôpital Saint-Louis, 1, Avenue Claude Vellefaux, 75010 Paris, France.

E-mail: laetitia.vercellino@aphp.fr

Published online Mar. 30, 2017.

COPYRIGHT (C 2017 by the Society of Nuclear Medicine and Molecular Imaging. de novo and postpolycythemia vera and postessential thrombocythemia myelofibrosis, which are long-term possible evolutions of polycythemia vera and essential thrombocythemia $(1,2)$.

The underlying pathologic process is the development of reticulin or collagen fibrosis in the bone marrow leading to impaired hematopoiesis that can be activated in unusual sites for adults including the peripheral skeleton (long bones) and extramedullary sites such as the spleen or the liver. The disease evolution is often characterized by the occurrence of cytopenias (mainly anemia), progressive splenomegaly, and constitutional symptoms, leading to a clearly impaired quality of life and reduced life expectancy, with an overall median survival of about 5-7 y $(3,4)$. Diagnosis relies on a set of criteria defined by the World Health Organization, including morphologic assessment of bone marrow histology, peripheral blood examination, and cytogenetic and molecular studies (5). The World Health Organization recognizes 4 grades of fibrosis ( $0-3$ ) based on reticulin density, collagen content, and osteosclerosis on the bone marrow biopsy (6). The bone marrow fibrosis grade may be of prognostic value, and may be useful for setting therapeutic strategy (7).

With the exception of allogeneic hematopoietic stem cell transplantation, treatments are only symptomatic (8). Ruxolitinib, a JAK1/JAK2 inhibitor, is currently the reference treatment for reduction of splenomegaly and improvement of constitutional symptoms, but there are still unmet needs especially for patients with anemia or thrombocytopenia (9-12).

Total-body imaging may be useful to evaluate the degree and extent of myelofibrosis and to identify extramedullary sites (their localizations and intensity), which are sources of disease complications depending on their location.

As underlined by Agool et al., there is a long story of bone marrow imaging with radionuclides (13). Before the development of PET technology with tracers assessing the metabolic activity, such as ${ }^{18} \mathrm{~F}$-FDG, or proliferative activity, such as $3^{\prime}$-deoxy- $3^{\prime}-{ }^{18} \mathrm{~F}$-fluorothymidine ( ${ }^{18} \mathrm{~F}$-FLT), several $\gamma$-emitting radionuclides enabled noninvasive assessment of bone marrow content and hematopoiesis. They could enable imaging of the reticuloendothelial system (now currently known as medullary stroma), erythroid precursor cell compartment, and myeloid (white cells) compartment.

The reference method of bone marrow scintigraphy (BMS) adopted in our department relies on combined imaging of the medullary stroma with ${ }^{99 \mathrm{~m}} \mathrm{Tc}$-nanocolloids and of the erythroid component of bone marrow with ${ }^{111} \mathrm{In}$-chloride coupled to serum 
transferrin (14-16). Nevertheless, BMS has some practical drawbacks: it requires 2 injections and 2 imaging acquisitions, $48 \mathrm{~h}$ apart. Moreover, patients who received transfusions may have a saturated transferrin. In these patients, ${ }^{111}$ In-chloride scintigraphy should be performed with heterologous plasma, which requires extraorganization and implies a theoretic risk of pathogen transmission. In our clinical department, BMS is still used as a routine technique to evaluate complex hematologic disorders such as myelofibrosis.

The use of PET imaging takes advantage of the availability of ${ }^{18}$ F-FLT as a specific tracer of cell proliferation and of highresolution images and quantification possibilities. ${ }^{18} \mathrm{~F}$-FLT PET may provide a potent and noninvasive tool for measuring global medullar activity and assessment of extramedullary hematopoiesis in several hematologic diseases such as myelodysplastic syndromes and myeloproliferative neoplasms (17). Furthermore, ${ }^{18}$ F-FLT PET has a noticeable practical advantage because PET images are obtained during the same examination, $1 \mathrm{~h}$ after ${ }^{18} \mathrm{~F}$ FLT injection. In addition, a PET quantitative approach may be of utmost interest in the evaluation of treatments and follow-up of these patients. However, few studies have been reported on this topic.

The present pilot study aimed to assess the feasibility of ${ }^{18} \mathrm{~F}-$ FLT PET imaging as an index of proliferative activity in myelofibrosis, which is a prerequisite for evaluation of diagnostic and prognostic values of the technique in this clinical setting.

\section{MATERIALS AND METHODS}

\section{Patients}

The patient population consisted of 15 patients with myelofibrosis strictly defined according to criteria of the World Health Organization (including primary myelofibrosis, postpolycythemia vera, and postessential thrombocythemia myelofibrosis) in the FLT-MF-2009 study (EudraCT no. 2009-016804-21). ${ }^{18}$ F-FLT PET was performed within the 3 mo after the inclusion. BMS was recommended, when possible.

The Committee on Ethics approved this study, and all subjects gave written informed consent.

\section{Bone Marrow Histopathology}

Bone marrow biopsy was performed on the posterior iliac crest and analyzed by an experienced hematopathologist. Morphologic study was made after hematoxylin and eosin staining, and the fibrosis grade was determined after Gomori's silver impregnation, using the European Consensus on grading of bone marrow fibrosis (grade $0-3$ ).

\section{BMS Acquisition}

The reticuloendothelial system imaging was performed 30 min after injection of ${ }^{99 \mathrm{~m}} \mathrm{Tc}$-nanocolloids (200 MBq). Afterward, patients were injected with plasma radiolabeled with ${ }^{111} \mathrm{In}$-chloride (100 MBq), and imaging was performed $48 \mathrm{~h}$ later. The absence of transferrin saturation had to be checked, and if transferrin saturation was present heterologous plasma was used for plasma labeling with ${ }^{111} \mathrm{In}$-chloride. Both planar acquisitions were obtained on a Symbia T2 (Siemens) $\gamma$-camera, ensuring an acquisition time of $15 \mathrm{~cm} / \mathrm{min}$.

\section{${ }^{18}$ F-FLT PET Acquisition}

PET/CT (computed tomodensitometry) images were obtained on a time-of-flight Gemini PET/CT (Philips), associating a fully 3-dimensional PET scanner with a 16-slice Brilliance CT scanner. Before PET acquisition, whole-body CT (from skull to toes) was performed with the following settings: $120 \mathrm{kv}, 100 \mathrm{~mA}$. An emission acquisition of the same field of view was performed $60 \mathrm{~min}$ after intravenous injection of
$3 \mathrm{MBq} / \mathrm{kg}$ of ${ }^{18} \mathrm{~F}$-FLT. An emission scan was acquired with an acquisition time of $1.45 \mathrm{~min}$ per bed position. Images were reconstructed with a blob ordered-subset time-of-flight list-mode iterative algorithm. CT images were used for attenuation correction of PET data.

\section{Image Analysis}

Two experienced nuclear physicians unaware of the patients' medical history conducted the visual analysis of all images. They reviewed all the image data separately, and whenever discordance occurred, consensus was obtained.

The qualitative analysis for ${ }^{18} \mathrm{~F}$-FLT PET and BMS was based on the interpretation criteria of BMS described in Rain et al., inspired by Mc Neil et al.: analysis of the axial skeleton and proximal limbs, assessment of extension on distal limbs, and involvement of spleen $(14,16)$. A 3-point visual scale was used to assess the intensity of uptake on both nanocolloid and transferrin imaging in each of the following anatomic regions: axial skeleton, upper and lower limbs (proximal and distal), liver, and spleen. For the axial skeleton and proximal limbs, the visual scale was 1 , markedly decreased; 2 , subnormal; and 3, normal uptake. For the distal limbs, the visual scale was 1 , no uptake; 2 , low uptake; and 3 , high uptake.

For the spleen, the visual scale was 1, physiologic uptake; 2 , increased uptake but less intense than in the liver; and 3, increased uptake equal or higher than in the liver.

This distribution helped to classify the patients according to the presumed severity of the disease and to describe the 3 patterns of tracer uptake tabulated at the bottom of Figure 1, which also shows an example of each pattern.

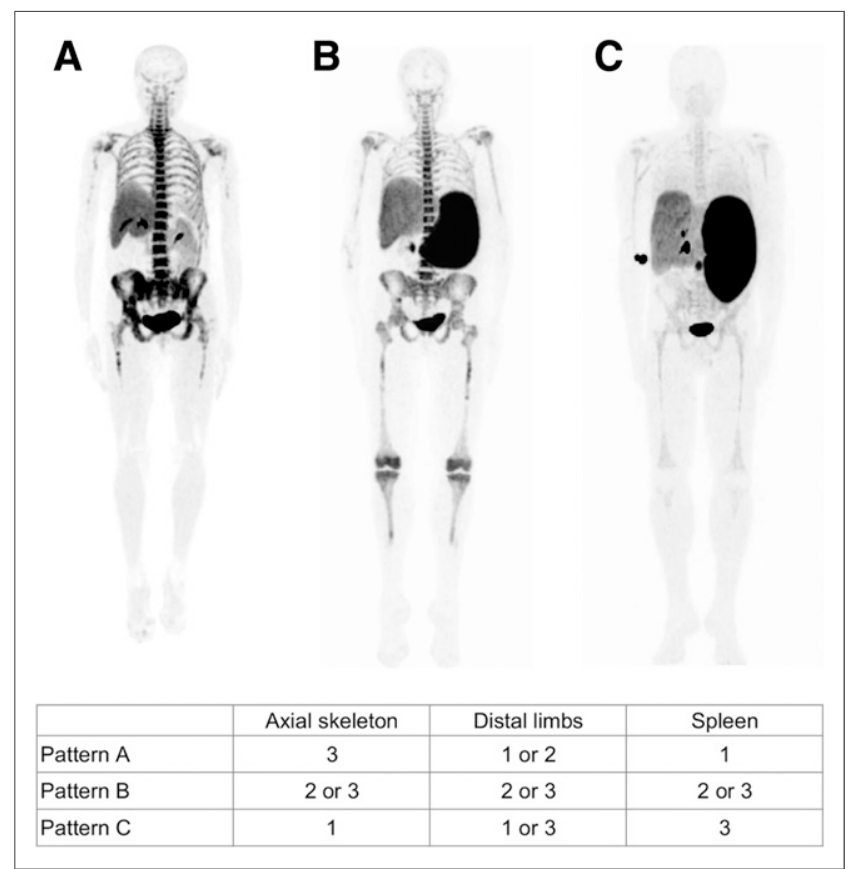

FIGURE 1. Patterns of ${ }^{18} \mathrm{~F}$-FLT distribution in myelofibrosis. Maximum-intensity projection corresponding to pattern $A(A)$, pattern $B(B)$, and pattern $C(C)$. Table underneath is summary of tracer distribution in each pattern. Pattern A showed a normal bone marrow activity in central skeleton, no or mild expansion to distal extremities, and normal splenic uptake. Pattern B displayed a normal or slightly decreased uptake in central skeleton but with marked expansion of bone marrow activity to distal extremities and intense uptake of tracer in spleen. Pattern C showed a marked reduced hematopoietic activity in central skeleton, with or without extension, and high uptake in spleen. 
TABLE 1

Patient Characteristics

\begin{tabular}{|c|c|c|c|c|c|c|c|}
\hline Patient no. & Age (y) & Type of MF & Grade of MF & $\begin{array}{l}\text { Previous } \\
\text { treatment }\end{array}$ & Hemoglobulin (g/dL) & Leukocytes (109/L) & Thrombocytes (109/L) \\
\hline 1 & 50 & post-PV & 2 & IFN & 14.6 & 8.60 & 285 \\
\hline 2 & 51 & PMF & 1 & IFN & 15.1 & 4.60 & 205 \\
\hline 3 & 52 & post-ET & 3 & IFN & 10.6 & 4.94 & 262 \\
\hline 4 & 59 & post-PV & 1 & IFN & 11.8 & 3.79 & 118 \\
\hline 5 & 73 & post-ET & 1 & UNT & 8.9 & 6.20 & 121 \\
\hline 6 & 53 & PMF & 3 & RUXO & 8.9 & 15.97 & 300 \\
\hline 7 & 51 & PMF & 3 & RUXO & 11.7 & 5.41 & 78 \\
\hline 8 & 70 & PMF & 2 & UNT & 7.4 & 22.54 & 130 \\
\hline 9 & 75 & post-PV & 1 & UNT & 14.9 & 8.193 & 146 \\
\hline 10 & 49 & PMF & 2 & UNT & 13.7 & 8.73 & 193 \\
\hline 11 & 69 & PMF & 1 & UNT & 13.2 & 5.39 & 246 \\
\hline 12 & 70 & post-ET & 2 & RUXO & 9.1 & 14.95 & 352 \\
\hline 13 & 69 & PMF & 3 & RUXO & 7.9 & 8.12 & 68 \\
\hline 14 & 65 & post-ET & 2 & UNT & 11.2 & 3.90 & 180 \\
\hline 15 & 71 & PMF & 2 & UNT & 11.3 & 14.38 & 215 \\
\hline
\end{tabular}

MF = myelofibrosis; post-PV = postpolycythemia vera; IFN = interferon; PMF = primary myelofibrosis; post-ET = postessential thrombocythemia; UNT = untreated; RUXO = ruxolitinib.

Moreover, a semiquantitative analysis by measuring $\mathrm{SUV}_{\max }$ on ${ }^{18}$ F-FLT PET images was performed in the same anatomic regions as qualitative analysis: axial skeleton (lumbar spine and iliac crest), proximal upper and lower limbs, distal upper and lower limbs, liver, and spleen.

\section{Statistical Analysis}

The quantitative distribution of each parameter was summarized by median and interquartile range (IQR) or mean $\pm \mathrm{SD}$. The analysis of factors associated with the intensity of the attachment of tumor ${ }^{18} \mathrm{~F}$ FLT marrow (using PET group and $\mathrm{SUV}_{\max }$ as the endpoints) was based on nonparametric tests, either Kruskal-Wallis or exact Fisher. Correlations between the different values of $\mathrm{SUV}_{\max }$ were assessed by the Spearman correlation coefficient. All statistical tests were 2-sided, with $P$ values of 0.05 or less denoting statistical significance. All analyses were performed using SAS 9.3 (SAS Inc.) or R 2.13.1 (http://www.R-project.org) statistical software.

\section{RESULTS}

\section{Patients}

Fifteen patients were included between April 2011 and July 2012. All of them underwent ${ }^{18}$ F-FLT PET, and all but 1 also underwent BMS. Patient characteristics are listed in Table 1. There were 9 men and 6 women. The median age was $65 \mathrm{y}$ (IQR, 51.5-70). The subtypes of myelofibrosis were primary myelofibrosis $(n=8)$, postessential thrombocythemia $(n=4)$, and postpolycythaemia vera myelofibrosis $(n=3)$. Therapies at the time of PET examination included interferon $(n=4)$ and ruxolitinib $(n=4) ; 7$ patients had no specific therapy for myelofibrosis.

\section{BMS}

Fourteen patients had BMS. Two patients needed heterologous plasma for the transferrin indium scintigraphy. Four patients presented with pattern $\mathrm{A}, 7$ with a pattern $\mathrm{B}$, and 3 with pattern
C, when transferrin imaging was considered. Of note, nanocolloid imaging is usually altered earlier than transferrin imaging in myelofibrosis, but it does not allow spleen analysis because of the intense physiologic splenic uptake of technetium-radiolabeled nanocolloids.

TABLE 2

BMS and ${ }^{18} \mathrm{~F}-\mathrm{FLT}$ Imaging Patterns and ${ }^{18} \mathrm{~F}-\mathrm{FLT}$ Semiquantitative Data

\begin{tabular}{lcccc}
\hline Patient no. & $\begin{array}{c}\text { BMS } \\
\text { pattern }\end{array}$ & $\begin{array}{c}18 \text { F-FLT } \\
\text { pattern }\end{array}$ & $\begin{array}{c}\text { Axial skeleton } \\
\left(\text { SUV }_{\text {max }}\right)\end{array}$ & $\begin{array}{c}\text { Spleen } \\
\left(\text { SUV }_{\text {max }}\right)\end{array}$ \\
\hline 1 & A & B & 16.0 & 10.0 \\
\hline 2 & A & A & 13.0 & 3.0 \\
\hline 3 & NA & B & 10.8 & 12.4 \\
\hline 4 & B & B & 18.4 & 13.1 \\
\hline 5 & C & C & 5.0 & 23.0 \\
\hline 6 & C & C & 2.0 & 10.4 \\
7 & C & C & 4.0 & 9.9 \\
\hline 8 & B & B & 15.0 & 12.4 \\
\hline 9 & B & B & 10.0 & 7.3 \\
\hline 10 & B & B & 12.8 & 13.2 \\
\hline 11 & A & A & 14.2 & 3.0 \\
12 & A & A & 13.9 & 3.5 \\
13 & B & B & 9.4 & 16.7 \\
\hline 14 & B & B & 10.3 & 13.8 \\
15 & B & B & 11.3 & 19.0 \\
\hline
\end{tabular}

$\mathrm{NA}=$ not applicable 


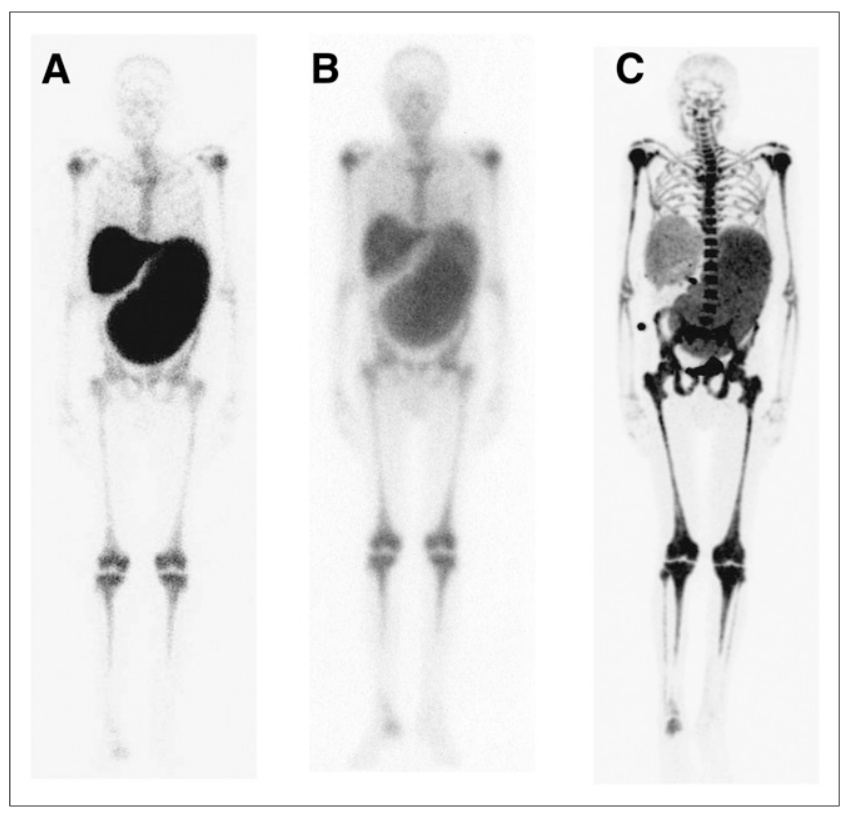

FIGURE 2. Example of comparison between BMS and ${ }^{18} \mathrm{~F}-\mathrm{FLT}$ PET (patient 9). (A) Anterior projection of ${ }^{99 \mathrm{mTC}} \mathrm{T}$-nanocolloid scintigraphy. (B) Anterior projection of ${ }^{111} \mathrm{In}$-chloride scintigraphy. (C) ${ }^{18} \mathrm{~F}$-FLT PET maximum-intensity projection.

\section{${ }^{18}$ F-FLT PET}

${ }^{18}$ F-FLT PET patterns and data are shown in Table 2. Three patients presented with pattern A, 9 with pattern $\mathrm{B}$, and 3 with pattern C.

In the study population, median $\mathrm{SUV}_{\max }$ was 11.3 (IQR, 9.714.05) for the axial skeleton, 7.6 (IQR, 5.5-10.45) for the right iliac crest, and 6.6 (IQR, 4.15-11.09) for the left iliac crest. For the spleen, the median $\mathrm{SUV}_{\max }$ was 12.4 (IQR, 8.6-13.5).

In patients with pattern $\mathrm{A}$, the mean $\mathrm{SUV}_{\max }$ was 13.7 in the axial skeleton and 3.2 in the spleen. In pattern $\mathrm{B}, \mathrm{SUV}_{\text {mean }}$ maximum was 12.5 in the axial skeleton and 13.1 in the spleen. Finally, in pattern $\mathrm{C}$ the $\mathrm{SUV}_{\text {mean }}$ maximum in the axial skeleton was 3.7 and 14.4 in the spleen.

The SUV of the axial skeleton was strongly correlated with that of the proximal limbs $(r=0.87, P<0.0001$ and $r=0.82, P=$ 0.0002 for upper and lower proximal limbs, respectively), while the SUV of distal limbs correlated somehow to each other ( $r=$ $0.59, P<0.0001)$. There was also a correlation between SUV in the spleen and liver $(r=0.68, P=0.0056)$. In addition, the $\mathrm{SUV}_{\max }$ of the right iliac crest was highly correlated to the $\mathrm{SUV}_{\text {max }}$ of left iliac crest $(r=0.88, P<0.0001)$.

\section{Comparison of Imaging Modalities}

In 13 of 14 patients who underwent the 2 imaging modalities, transferrin imaging and ${ }^{18}$ F-FLT PET yielded the same distribution pattern, as illustrated by Figure 2. In patient 1, although BMS was inconclusive for splenic involvement, PET showed clearly increased splenic uptake. Thanks to a much better definition of images, qualitative analysis of ${ }^{18}$ F-FLT PET images was easier than that of BMS. Moreover, semiquantitative analysis of PET data was also helpful for interpretation, by providing objective information.

\section{Comparison of PET Data and Bone Marrow Histology}

A significant correlation was observed between the uptake of ${ }^{18}$ F-FLT assessed with $\mathrm{SUV}_{\max }$ and histologic grade determined on bone marrow biopsy (Table 3 ). $\mathrm{SUV}_{\max }$ measured in the spine and proximal limbs appeared to be correlated with the grade of fibrosis. In grade 3 myelofibrosis, uptake in the axial skeleton and proximal limbs was significantly lower than in grade 1 and 2 myelofibrosis $(P=0.019$ for axial skeleton, $P=0.016$ for proximal upper limbs, and $P=0.019$ for proximal lower limbs) as shown in Figure 3.

However, no statistical correlation was found between the visual patterns described with ${ }^{18} \mathrm{~F}$-FLT PET and bone marrow histology.

\section{DISCUSSION}

The present study shows the feasibility and the potential relevance of ${ }^{18} \mathrm{~F}$-FLT PET in assessing dysregulated proliferation of myeloid cells in myelofibrosis. Similar findings have been reported in a series of 3 patients with myelofibrosis: Agool et al. observed a tracer distribution combining decreased tracer uptake in axial bone marrow with increased uptake in the spleen (17). Nevertheless, we observed additional distribution patterns in our study such as a characteristic pattern in 3 patients who showed almost absence of tracer uptake in axial skeleton and very high uptake in the spleen, suggesting a shift in hematopoietic activity as expected in advanced myelofibrosis. In contrast, 3 other patients did not show any pathologic tracer uptake in the spleen, suggesting

TABLE 3

Comparison Between Histologic Grade and ${ }^{18} \mathrm{~F}-\mathrm{FLT}$ Semiquantitative Data

\begin{tabular}{|c|c|c|c|c|c|}
\hline Parameter & Grade $1(n=5)$ & Grade $2(n=6)$ & Grade $3(n=4)$ & $P(1$ vs. 2 vs. 3$)$ & $P(1+2$ vs. 3$)$ \\
\hline Axial skeleton & $12.1 \pm 5.0^{\star}$ & $13.2 \pm 2.2$ & $6.5 \pm 4.2$ & 0.058 & 0.019 \\
\hline Proximal upper limbs & $10.0 \pm 4.1$ & $8.2 \pm 4.2$ & $3.8 \pm 1.9$ & 0.044 & 0.016 \\
\hline Distal upper limbs & $1.6 \pm 1.1$ & $1.0 \pm 0.7$ & $1.2 \pm 0.9$ & 0.52 & 0.95 \\
\hline Proximal lower limbs & $8.9 \pm 3.3$ & $8.2 \pm 4.4$ & $3.6 \pm 1.8$ & 0.053 & 0.019 \\
\hline Distal lower limbs & $1.5 \pm 2.8$ & $1.7 \pm 2.0$ & $2.2 \pm 2.4$ & 0.41 & 0.95 \\
\hline Right iliac crest & $8.3 \pm 3.9$ & $9.6 \pm 3.8$ & $4 \pm 2.1$ & 0.083 & 0.026 \\
\hline Left iliac crest & $8.2 \pm 5.5$ & $9.6 \pm 3.5$ & $3.9 \pm 2.2$ & 0.087 & 0.037 \\
\hline Spleen & $9.9 \pm 8.4$ & $12.0 \pm 5.1$ & $12.4 \pm 3.1$ & 0.60 & 0.84 \\
\hline
\end{tabular}




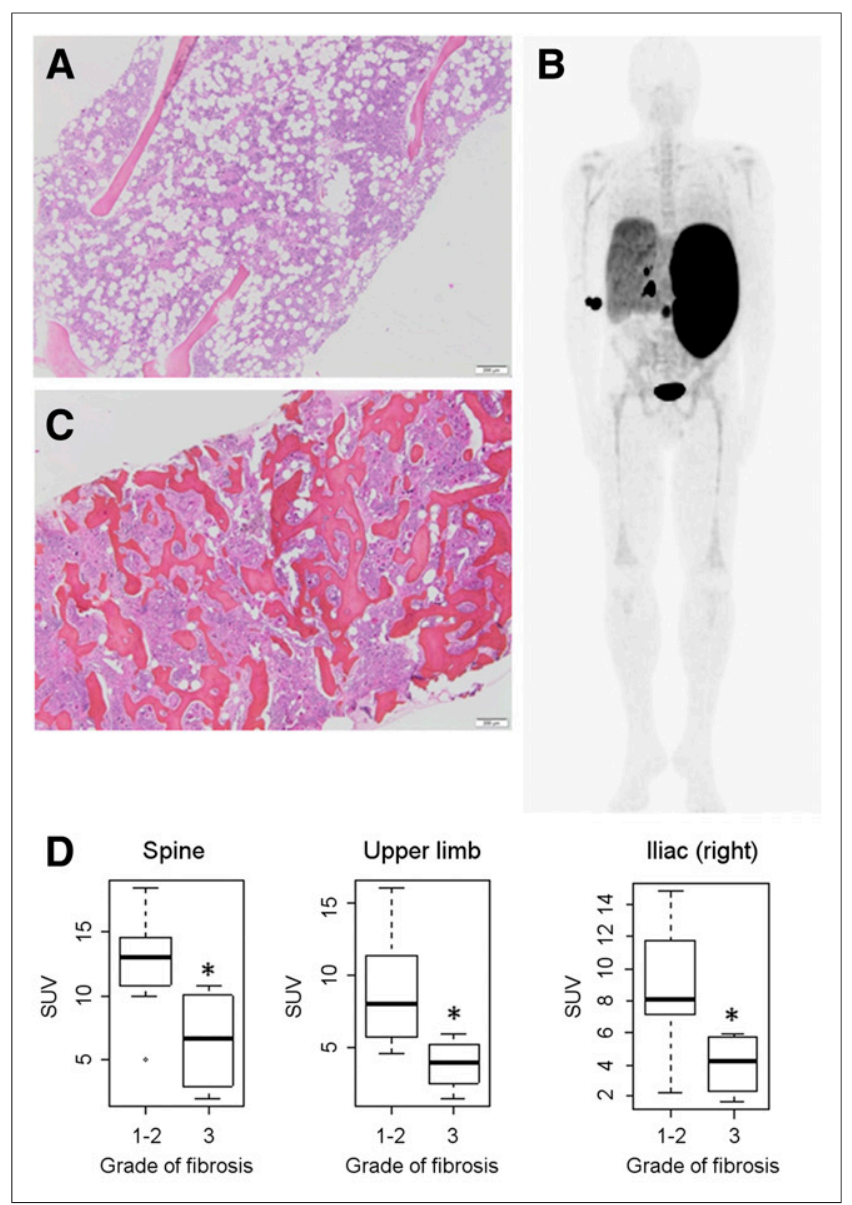

FIGURE 3. Correlation of fibrosis grade and ${ }^{18} \mathrm{~F}-\mathrm{FLT}$ uptake in axial skeleton. (A) Normal bone marrow biopsy with no fibrosis (hematoxylin and eosin, $\times 40$ ). (B) ${ }^{18} \mathrm{~F}$-FLT PET of patient 6 , showing low uptake in axial myelofibrosis $\left(\mathrm{SUV}_{\max }=2\right.$ in axial skeleton, 1.7 in right iliac crest, and 1.6 in left iliac crest). (C) Bone marrow biopsy of patient 6 showing grade 3 fibrosis, with osteosclerosis according to European Consensus on grading of bone marrow fibrosis (hematoxylin and eosin, $\times 40$ ). (D) Distribution of SUV max in spine, proximal upper limbs, and right iliac crest for patients depending on histologic grade of fibrosis. SUV $\mathrm{max}_{\max }$ in grade 3 fibrosis patients is lower than in patients with grade 1 or 2 fibrosis. ${ }^{*}$ Translates to significant difference between groups.

the absence of extramedullar hematopoiesis, as observed in early stages of the disease.

Furthermore, our study provides a comparison between PET and BMS, the current reference nuclear medicine imaging modality for this disease. ${ }^{18} \mathrm{~F}$-FLT PET is much more convenient than BMS for bone marrow imaging because ${ }^{18} \mathrm{~F}$-FLT PET requires a shorter acquisition procedure, and BMS needs 2 imaging procedures on 2 separate days. ${ }^{18}$ F-FLT PET is also easier to interpret than BMS because of better image quality and most importantly allows semiquantitative analysis. Indeed, $\mathrm{SUV}_{\max }$ measurements obtained in different sites provide helpful information for interpretation of a patient's status. The detection of the presence of extramedullary hematopoiesis, usually observed in the spleen, is of critical importance.

If BMS, with the additional use of hybrid imaging with SPECT coupled to CT, remains a valuable diagnostic procedure, its wider use is hindered by the complexity and time inherent to this imaging modality (18).
An important new finding of our study is the correlation between $\mathrm{SUV}_{\max }$ in the axial skeleton and proximal limbs and the grade of fibrosis. Patients with grade 3 fibrosis had a significantly lower tracer uptake than patients with grade 1 or 2 fibrosis, suggesting that ${ }^{18} \mathrm{~F}$-FLT PET data could be a noninvasive tool for globally assessing the severity of reticulin fibrosis in patients with myelofibrosis.

Visual analysis of BMS and ${ }^{18}$ F-FLT PET according to 3 different patterns of tracer uptake could help distinguish subgroups of patients, ranging from a subnormal distribution to a highly altered uptake of the tracer with low uptake of ${ }^{18}$ F-FLT in the axial skeleton and high uptake in the spleen. Nevertheless, in contrast to what was observed with quantitative data, no statistical correlation was found between the patterns defined by visual analyses of ${ }^{18}$ F-FLT PET images and the grade of fibrosis. Although this might be due to the limited number of patients included in this pilot study, it is likely that the definition of 3 different patterns might not render all the subtle information provided by the quantitative index of uptake (SUV). Several scoring systems have been proposed for the classification of bone marrow uptake. Kniseley reported a 5-point scale to analyze radiolabeled nanocolloid distribution, from normal bone marrow uptake to loss of marrow uptake. As mentioned by the author himself, this classification did not take into account liver and spleen uptake, which is a physiologic pattern with this tracer (19). However, as also suggested by the author, typing the image of patients could prevent us from fully interpreting the imaging data and make us miss some information. A similar explanation may be also applicable in our study and explain why our patterns did not describe the complexity of what was observed.

Thanks to its characteristics, especially its potential of imaging quantification, ${ }^{18}$ F-FLT PET may be a relevant tool in assessing the efficacy of the new therapeutic drugs available for myelofibrosis. This should be confirmed in a larger study. Indeed, ${ }^{18} \mathrm{~F}-\mathrm{FLT}$ PET has been used previously to follow the response to anticancer therapy, either in solid neoplasms or in leukemias. Moreover, it has also been recently proposed as a tool to evaluate the impact of chemotherapy on normal bone marrow, due to its potential ability to assess both therapeutic response and hematologic toxicity in a single examination. In a pilot study, Vanderhoek et al. proposed ${ }^{18}$ F-FLT PET whole-body imaging as a surrogate marker of early response to treatment in patients with acute myeloid leukemia receiving induction chemotherapy because it allows a noninvasive evaluation of the whole-bone marrow, free of the sampling effect of bone marrow biopsy (20). Prior data have also suggested a potential role of ${ }^{18} \mathrm{~F}$-FLT PET imaging in identifying bone marrow toxicity due to chemo- and radiotherapy (21). McGuire et al. demonstrated a correlation between reduction of ${ }^{18} \mathrm{~F}$-FLT uptake during radiotherapy and the radiation dose received by the bone marrow (22).

The ability of ${ }^{18}$ F-FLT PET imaging to provide longitudinal information of bone marrow activity or spleen activation was also suggested in patients receiving concurrent radiochemotherapy for non-small cell lung cancer, in whom a decrease of ${ }^{18}$ F-FLT uptake was found in bone marrow before the decrease in neutrophil counts, whereas a decreased uptake followed by a rebound was observed in the spleen (23).

Other studies, based on the hypothesis that myelofibrosis presents an inflammatory component, reported the use of ${ }^{18} \mathrm{~F}$ FDG PET to evaluate patients with myelofibrosis (24). An inverse correlation was found between ${ }^{18} \mathrm{~F}$-FDG uptake and the grade of 
fibrosis, with a more intense and widespread ${ }^{18} \mathrm{~F}-\mathrm{FDG}$ uptake (in skeleton and spleen) being present at earlier stage than later stage due to differences in extent of inflammation and fibrosis in the bone marrow. However, ${ }^{18}$ F-FLT PET has the advantage of assessing directly the myeloproliferative activity, irrespective of the presence of inflammation. It can describe accurately the distribution of active bone marrow, through the evaluation of the decrease of activity in the axial skeleton, extension of active bone marrow with recruitment of fetal sites (skull, distal limbs) of hematopoiesis, and the myeloid transformation of the spleen. ${ }^{18}$ F-FLT PET then yields, in a rather objective manner, information about the severity of the disease. If performed at diagnosis, or before the onset of specific therapy, baseline ${ }^{18}$ F-FLT PET may serve as reference imaging to later evaluate the efficacy of therapies.

Thus, ${ }^{18}$ F-FLT PET imaging promises to perform noninvasively follow-up studies in myelofibrosis patients to assess the impact of new therapeutic intervention on the dynamics of bone marrow and extramedullary hematopoietic activity. New therapeutic agents for myelofibrosis have emerged recently, such as JAK inhibitors. These drugs are efficient in reducing the spleen size but do not always restore normal bone marrow function. ${ }^{18}$ F-FLT PET could be of great interest to assess the effect of such therapies on the evolution of the fibrosis and the proliferative activity of bone marrow.

\section{CONCLUSION}

${ }^{18}$ F-FLT PET may be a useful new tool for the evaluation of patients with myelofibrosis. Distinct patterns of ${ }^{18} \mathrm{~F}$-FLT uptake may be of value in the diagnosis and classification of myelofibrosis patients. The correlation of quantitative data of ${ }^{18} \mathrm{~F}-\mathrm{FLT}$ PET imaging with histology suggests the potential of this noninvasive technique to assess myelofibrosis evolution during therapy, as an alternative to sequential follow-up biopsies.

\section{DISCLOSURE}

Advanced Accelerator Applications (AAA, France) provided the radiotracer ${ }^{18} \mathrm{~F}$-FLT free of charge. No other potential conflict of interest relevant to this article was reported.

\section{ACKNOWLEDGMENTS}

We thank Antoine Martineau, $\mathrm{PhD}$, for helping us to prepare the figures.

\section{REFERENCES}

1. Tefferi A. Myelofibrosis with myeloid metaplasia. N Engl J Med. 2000;342: 1255-1265.

2. Tefferi A, Thiele J, Orazi A, et al. Proposals and rationale for revision of the World Health Organization diagnostic criteria for polycythemia vera, essential thrombocythemia, and primary myelofibrosis: recommendations from an ad hoc international expert panel. Blood. 2007;110:1092-1097.
3. Barbui T, Barosi G, Birgegard G, et al. Philadelphia-negative classical myeloproliferative neoplasms: critical concepts and management recommendations from European LeukemiaNet. J Clin Oncol. 2011;29:761-770.

4. Tefferi A. Primary myelofibrosis: 2013 update on diagnosis, risk-stratification, and management. Am J Hematol. 2013;88:141-150.

5. Arber DA, Orazi A, Hasserjian R, et al. The 2016 revision to the World Health Organization classification of myeloid neoplasms and acute leukemia. Blood. 2016;127:2391-2405.

6. Thiele J, Kvasnicka HM, Facchetti F, Franco V, van der Walt J, Orazi A. European consensus on grading bone marrow fibrosis and assessment of cellularity. Haematologica. 2005;90:1128-1132.

7. Gianelli U, Vener C, Bossi A, et al. The European consensus on grading of bone marrow fibrosis allows a better prognostication of patients with primary myelofibrosis. Mod Pathol. 2012;25:1193-1202.

8. Mughal TI, Vaddi K, Sarlis NJ, Verstovsek S. Myelofibrosis-associated complications: pathogenesis, clinical manifestations, and effects on outcomes. Int J Gen Med. 2014;7:89-101.

9. Harrison C, Kiladjian JJ, Al-Ali HK, et al. JAK inhibition with ruxolitinib versus best available therapy for myelofibrosis. N Engl J Med. 2012;366:787-798.

10. Verstovsek S, Mesa RA, Gotlib J, et al. A double-blind, placebo-controlled trial of ruxolitinib for myelofibrosis. N Engl J Med. 2012;366:799-807.

11. Verstovsek S, Mesa RA, Gotlib J, et al. The clinical benefit of ruxolitinib across patient subgroups: analysis of a placebo-controlled, phase III study in patients with myelofibrosis. Br J Haematol. 2013;161:508-516.

12. Verstovsek S, Mesa RA, Gotlib J, et al. Efficacy, safety and survival with ruxolitinib in patients with myelofibrosis: results of a median 2-year follow-up of COMFORT-I. Haematologica. 2013;98:1865-1871.

13. Agool A, Glaudemans AW, Boersma HH, Dierckx RA, Vellenga E, Slart RH. Radionuclide imaging of bone marrow disorders. Eur J Nucl Med Mol Imaging. 2011;38:166-178.

14. McNeil BJ, Holman BL, Button LN, Rosenthal DS. Use of indium chloride scintigraphy in patients with myelofibrosis. J Nucl Med. 1974;15:647-651.

15. Sayle BA, Helmer RE, 3rd, Birdsong BA, Balachandran S, Gardner FH. Bonemarrow imaging with indium-111 chloride in aplastic anemia and myelofibrosis: concise communication. J Nucl Med. 1982;23:121-125.

16. Rain JD, Najean Y, Billotey C. Bone marrow scintigraphy as a useful method for estimating the physiological status of bone marrow and spleen in polycythaemia vera. Leuk Lymphoma. 1996;22:105-110.

17. Agool A, Schot BW, Jager PL, Vellenga E. ${ }^{18}$ F-FLT PET in hematologic disorders: a novel technique to analyze the bone marrow compartment. J Nucl Med. 2006;47:1592-1598.

18. Ojeda-Uribe M, Morel O, Ungureanu C, Desterke C, Le Bousse-Kerdilès M-C, Boulahdour H. Assessment of sites of marrow and extramedullary hematopoiesis by hybrid imaging in primary myelofibrosis patients. Cancer Med. 2016;5:23782384 .

19. Kniseley RM. Marrow studies with radiocolloids. Semin Nucl Med. 1972;2: 71-85.

20. Vanderhoek M, Juckett MB, Perlman SB, Nickles RJ, Jeraj R. Early assessment of treatment response in patients with AML using $\left[{ }^{18}\right.$ F]FLT PET imaging. Leuk Res. 2011;35:310-316.

21. Agool A, Slart RH, Thorp KK, et al. Effect of radiotherapy and chemotherapy on bone marrow activity: a ${ }^{18}$ F-FLT-PET study. Nucl Med Commun. 2011;32:17-22.

22. McGuire SM, Menda Y, Boles Ponto LL, Gross B, Buatti J, Bayouth JE. 3'-deoxy-3' $\left[{ }^{18} \mathrm{~F}\right]$ fluorothymidine PET quantification of bone marrow response to radiation dose. Int J Radiat Oncol Biol Phys. 2011;81:888-893.

23. Leimgruber A, Moller A, Everitt SJ, et al. Effect of platinum-based chemoradiotherapy on cellular proliferation in bone marrow and spleen, estimated by ${ }^{18} \mathrm{~F}$ FLT PET/CT in patients with locally advanced non-small cell lung cancer. $J$ Nucl Med. 2014;55:1075-1080.

24. Derlin T, Alchalby H, Bannas P, et al. Assessment of bone marrow inflammation in patients with myelofibrosis: an ${ }^{18} \mathrm{~F}$-fluorodeoxyglucose PET/CT study. Eur $J$ Nucl Med Mol Imaging. 2015;42:696-705. 